Invited Paper

\title{
Advanced electrodynamic mechanisms for the nanoscale control of light by light
}

\author{
David L. Andrews,* Jamie M. Leeder, and David S. Bradshaw \\ School of Chemistry, University of East Anglia, Norwich Research Park, Norwich NR4 7TJ, U. K.
}

\begin{abstract}
A wide range of mechanisms is available for achieving rapid optical responsivity in material components. Amongst them, some of the most promising for potential device applications are those associated with an ultrafast response and a short cycle time. These twin criteria for photoresponsive action substantially favor optical, over most other, forms of response such as those fundamentally associated with photothermal, photochemical or optomechanical processes. The engagement of nonlinear mechanisms to actively control the characteristics of optical materials is not new. Indeed, it has been known for over fifty years that polarization effects of this nature occur in the optical Kerr effect - although in fluid media the involvement of a molecular reorientation mechanism leads to a significant response time. It has more recently emerged that there are other, less familiar forms of optical nonlinearity that can provide a means for one beam of light to instantly influence another. In particular, major material properties such as absorptivity or emissivity can be subjected to instant and highly localized control by the transmission of light with an off-resonant wavelength. This presentation introduces and compares the key electrodynamic mechanisms, discussing the features that suggest the most attractive possibilities for exploitation. The most significant of such mechanistic features include the off-resonant activation of optical emission, the control of excited-state lifetimes, the access of dark states, the inhibition or re-direction of exciton migration, and a coupling of stimulated emission with coherent scattering. It is shown that these offer a variety of new possibilities for ultrafast optical switching and transistor action, ultimately providing all-optical control with nanoscale precision.
\end{abstract}

Keywords: non-linear optics, all-optical processes, quantum electrodynamics, photo-active devices, optical transistor, stimulated emission, nanophotonics, energy transfer, ac Kerr effect, optical switching.

\section{INTRODUCTION}

Using quantum electrodynamics (QED) as a theoretical framework, a number of novel developments have emerged in relation to controlling light with light - especially in relation to emission. Such advances relate loosely to the wellknown ac Kerr effect, in which an applied electromagnetic field induces a change in the refractive index of a material with any light passing through it also altered, or by the fact that the throughput of a laser beam into a system may produce stimulated emission when the laser frequency matches the emission energy - a phenomenon with many applications, as for example in the recent Nobel Prize winning technique known as stimulated emission depletion spectroscopy. ${ }^{1-6}$ However, outside of stimulated emission, it has emerged that a moderately intense, off-resonant laser beam may significantly alter the rate and intensity of emission. ${ }^{7-10}$ Under these circumstances, the probe laser essentially confers optical nonlinearity onto the emission - and consequently, each excited-state lifetime is appreciably modified. Indeed, in principle a very long lifetime (essentially forbidden) transition could be activated. Although certain analogies might be drawn with the enhancement of optical emission through its coupling with plasmonic surfaces, ${ }^{11-20}$ the present development modifies spontaneous emission through direct interaction with the oscillating electric field of throughput radiation, without the need of any surface. Related to the control of emission is the coupling of stimulated emission with coherent scattering, which may lead to optical transistor action, and the population of dark states by the control of light

Active Photonic Materials VII, edited by Ganapathi S. Subramania, Stavroula Foteinopoulou, Proc. of SPIE Vol. 9546, 954600 - (C) 2015 SPIE · CCC code: 0277-786X/15/\$18 · doi: 10.1117/12.2190191 
absorption. The following account will address this along with other associated topics, including all-optical switching based on the inhibition of exciton migration.

\section{OPTICAL CONTROL OF LIGHT EMISSION}

The theoretical framework for understanding the control of light by light is QED. In such a theory, light-matter interactions are described, using perturbation theory, by transition probabilities. These factors are accordingly addressed through quantum probability amplitudes that define the coupling between initial and final system states, through any possible combination of intermediate state transitions. The experimentally measurable rate, $\Gamma$, of a given process can then be determined through Fermi's Rule, which is expressed as follows:

$$
\Gamma=\frac{2 \pi \rho}{\hbar}|M|^{2}
$$

Here, $\rho$ is the density of states and $M$ is the quantum amplitude explicitly given by;

$$
M=\left\langle F\left|H_{\text {int }}\right| I\right\rangle+\sum_{R, S} \frac{\left\langle F\left|H_{\text {int }}\right| S\right\rangle\left\langle S\left|H_{\text {int }}\right| R\right\rangle\left\langle R\left|H_{\text {int }}\right| I\right\rangle}{\left(E_{I}-E_{R}\right)\left(E_{I}-E_{S}\right)}+\ldots
$$

where $H_{\text {int }}$ is the interaction Hamiltonian of the system, $I$ and $F$ are the respective initial and final system states $(R, S$ are intermediate states) and $E$ is the energy of a state denoted by its subscript. Here, only the odd terms are given, since these are the ones that are relevant in absorption and emission mechanisms. In fact, it is usually only the first term that is important, with the second term essentially denoting self-energy corrections. However, on application of an off-resonant laser beam of sufficient intensity - namely, a beam whose wavelength lies within a transparent region of the irradiated material - the second term becomes significant (as will be shown). In the present account we now concentrate on emission processes, noting that the counterpart analysis of absorption mechanisms follows along similar lines. ${ }^{21-23}$

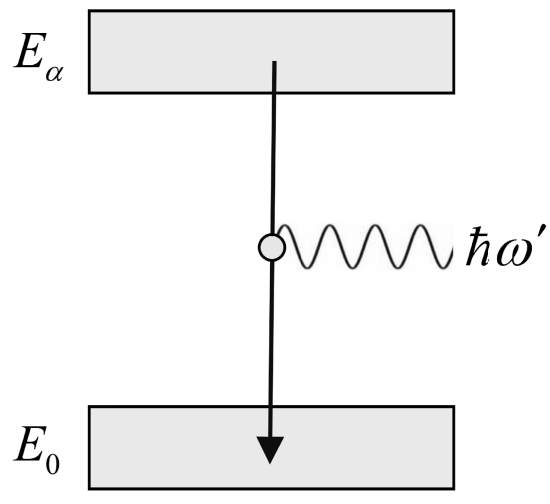

(a)

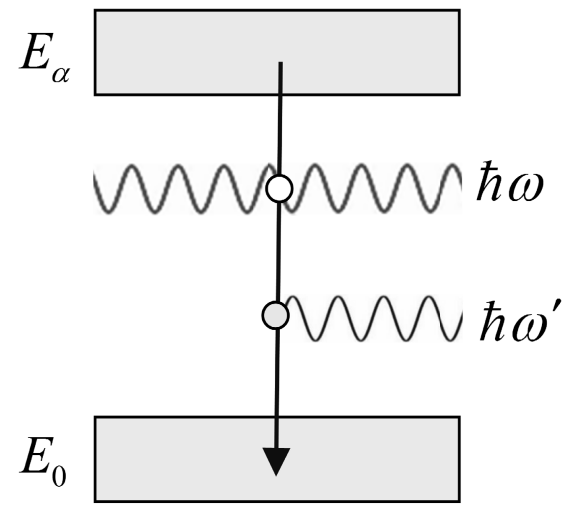

(b)

Figure 1. Energy level representation for: $(a)$ spontaneous and (b) nonlinear coupling mechanisms for light emission. Electronic states (and their vibrational manifolds) are signified by boxes, where $E_{0}$ and $E_{\alpha}$ are correspondingly the ground and excited molecular states. Wavy lines indicate photon propagation and the vertical arrow is a decay transition due to the emission. The emitted light has energy defined by $\hbar \omega^{\prime}$, while photons of the off-resonant laser beam are of energy $\hbar \omega$. The grey dot symbolizes a single matterradiation interaction and the white dot represents two such interactions (i.e. elastic forward scattering). 
In general the emission intensity, $I^{\prime}$, is found by multiplying the Fermi Rule of equation (1) by the energy of an emitted photon $\hbar \omega^{\prime} \equiv \hbar c k^{\prime}$; this signifies the emission signal that follows relaxation from an excited state. The net intensity is found from $I^{\prime}=2 \pi \rho c k^{\prime}|M|^{2}$, where $M$ includes the first term of equation (2) - which corresponds to conventional emission as illustrated by Figure 1 $(a)$ - and the second term is relevant when the probe beam is applied as shown by Figure 1(b); higher-order terms contribute negligibly. As determined elsewhere, ${ }^{9}$ the following general result is discovered;

$$
\begin{aligned}
I^{\prime} & =\left(\frac{c k^{\prime 4}}{8 \pi^{2} \varepsilon_{0}}\right)\left[e_{i}^{\prime} e_{j}^{\prime} \mu_{i}^{0 \alpha} \bar{\mu}_{j}^{0 \alpha}+\left(I / c \varepsilon_{0}\right) e_{i} e_{j} e_{k}^{\prime} e_{l}^{\prime} \chi_{i j k}^{0 \alpha}\left(\omega^{\prime} ;-\omega, \omega\right) \bar{\mu}_{l}^{0 \alpha}\right. \\
& \left.+\left(I^{2} / 4 c^{2} \varepsilon_{0}^{2}\right) e_{i} e_{j} e_{k}^{\prime} e_{l} e_{m} e_{n}^{\prime} \chi_{i j k}^{0 \alpha}\left(\omega^{\prime} ;-\omega, \omega\right) \bar{\chi}_{l m n}^{0 \alpha}\left(\omega^{\prime} ;-\omega, \omega\right)\right],
\end{aligned}
$$

where $I$ is the irradiance of the laser probe, $\chi_{i j k}^{0 \alpha}\left(\omega^{\prime} ;-\omega, \omega\right)$ is the nonlinear transition hyperpolarizability tensor and $\mathbf{e}$ represents the polarization vector of the input photons with energy $\hbar c k$. The first term corresponds to spontaneous emission, intrinsic to the system and independent of the probe laser beam, while the last term signifies a coupling of the elastically forward-scattered probe beam with the emission. The middle term, linear in $I$, signifies a quantum interference of these two concurrent processes. In general, it is assumed that the leading term in equation (3) is non-zero and the second one is the leading correction - this leads to interesting fluorescence anisotropic effects. ${ }^{7,10}$ However, there are circumstances in which the final term (which corresponds to nonlinear optical response) alone provides the emission, i.e. when the first and second terms of equation (3) are null. This will now be explored.

Consider, for instance, a system where the electronic population is efficiently transferred to a state $\alpha$ that would normally decay non-radiatively, where transitions from $\alpha$ to 0 are weak or entirely precluded - as, for example, through inherent geometric or symmetry constraints. In such a situation, terms in equation (3) that feature the transition dipole $\mu^{0 \alpha}$ do not contribute: radiative emission only occurs in a response to the off-resonant throughput beam, in a three-photon allowed transition. Such a configuration may indeed provide the basis for an all-optical switch, whose operation would be as follows: (i) a molecule is indirectly excited to a 'dark' state (i.e. one whose direct excitation from the ground state is forbidden); (ii) precluded by the one-photon dipole selection rules, emission from the dark state occurs on application of the probe beam only; (iii) this activation of the emission occurs for electronic transitions that are three-photon allowed, but single-photon forbidden. To summarize, switching action occurs since the throughput and absence of the input laser results in activation and deactivation of the emission, respectively - namely, the nanoscale control of light by light. Practically, it will be necessary for the radiation to be delivered in a pulse whose duration and delay, both with respect to the initial excitation, are sufficiently short that it can engage with the system before there is significant non-radiative dissipation of the excited state.

In addition, it is interesting that indirect excitation to a dark state may be possible by a system analogous to lightcontrolled emission. In terms of quantum electrodynamics, light absorption is simply emission reversed so that, as a result, similar equations will arise ${ }^{22}$ the mechanisms corresponding to such a case are presented in Figure 2 . It is thus possible for electronic states that are usually inaccessible (i.e. electronic transition that are one-photon forbidden) to become reachable by the three-photon mechanism. It should also be noted that a similar all-optical switching process has previously been proposed based on resonance energy transfer. ${ }^{24,25}$ 


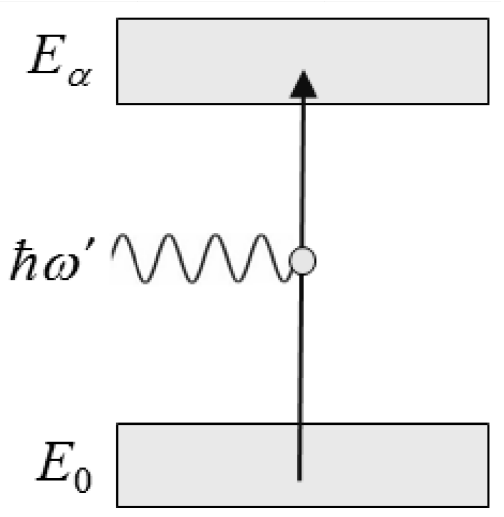

(a)

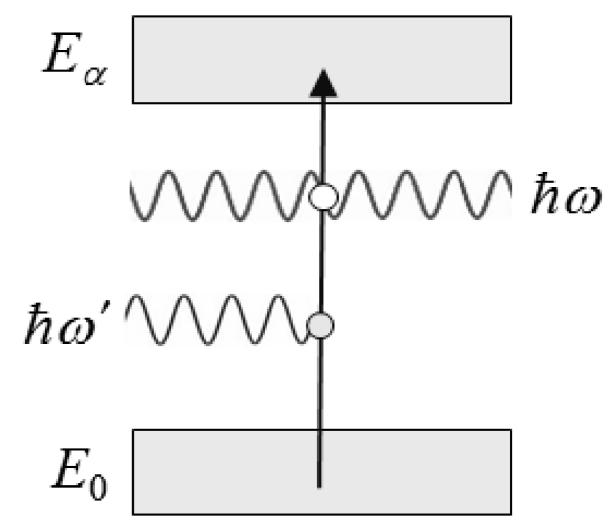

(b)

Figure 2. Energy level representation for: $(a)$ one-photon absorption and $(b)$ a nonlinear coupling process involving light absorption. The absorbed photon has an energy $\hbar \omega^{\prime}$, and the vertical arrow is an excitation transition.

\section{OPTICAL TRANSISTOR ACTION}

In this section we present a potential optical transistor device using our novel light by light scheme as a foundation. ${ }^{8}$ Our proposal is separate from others previously suggested, including systems based on polaritons, ${ }^{26,27}$ the optical Kerr effect, ${ }^{28,29}$ electromagnetically induced transparency, ${ }^{30-32}$ saturated absorption, ${ }^{33}$ and beam pattern rotation by application of a signal beam. ${ }^{34}$ A single-photon transistor has also been reported, which works on the principle that a 'dipole blockade' occurs in situations when a Group I atom in an ultracold gas is in a Rydberg excited state - namely, following initial excitation of an atom by light, further absorption by atoms in close proximity is unlikely. Hence, the sustained excitation of long-lived high Rydberg $p$ states produces a saturation effect that is manifest in an essential transparency to the pump radiation. However, by controlling the relative energy positioning of the Rydberg $s$ and $p$ levels through a Stark shift, ${ }^{35}$ it is possible to bring atoms that are in close proximity into a Förster resonance, transferring excitation into the $s$ levels and so depopulating the $p$ levels. The result is a desaturation that shows up as a dramatic increase in absorption of the pump radiation, signifying a blockade in its transmission. ${ }^{36}$

Let us consider a typical three-level laser system optically pumped within a microcavity. The kinetics of emission are primarily determined by a pump rate $R_{p}$ driving a population from the ground state $E_{0}$ into a metastable upper level $E_{2}$, with stimulated emission occurring as $E_{2}$ decays to $E_{1}$, and ultrafast relaxation from $E_{1}$ (Figure 3). Following Siegman, ${ }^{37}$ the rate equations for the temporal behavior of the cavity photon number, $n$, and the $E_{2}$ population, $N_{2}$, are as follows:

$$
\begin{gathered}
\frac{d n}{d t}=(n+1) K N_{2}-\gamma_{c} n, \\
\frac{d N_{2}}{d t}=R_{p}-n K N_{2}-\gamma_{2} N_{2}
\end{gathered}
$$

Here, the population of $E_{1}$ is assumed to be negligible; $K$ denotes the coupling coefficient for the laser transition, and $\gamma_{c}$ and $\gamma_{2}$ signify the cavity and population decay rates, respectively. Under steady-state conditions, equations (4) and (5) are combined to produce; 


$$
n=\frac{R_{p}-g \gamma_{c} p+\left[\left(R_{p}-g \gamma_{c} p\right)^{2}+4 \gamma_{c} R_{p}\right]^{\frac{1}{2}}}{2 \gamma_{c}} .
$$

where $g=\gamma_{2} / \gamma_{\mathrm{rad}}$ and $K=\gamma_{\mathrm{rad}} / p$ (in which $\gamma_{\mathrm{rad}}$ is the radiative decay rate and $p$ is the number of resonant cavity modes). The relaxation from $E_{2}$ into $E_{1}$ is not entirely radiative, i.e. $\gamma_{2} \neq \gamma_{\text {rad }}$, since non-radiative relaxation processes contribute to the decay. For present calculational purposes, given that the level $E_{2}$ decay rate $\gamma_{2}$ subsumes - but is dominated by - the rate of radiative decay, we may assume that $g=5 / 4$ in the absence of the off-resonant probe. From equation (6), employing typical values $p=10^{10}$ and $\gamma_{c}=10^{8} \mathrm{~s}^{-1}$, the familiar vertical climb in cavity photons at laser threshold emerges, as graphically illustrated by Figure 4 (solid line).

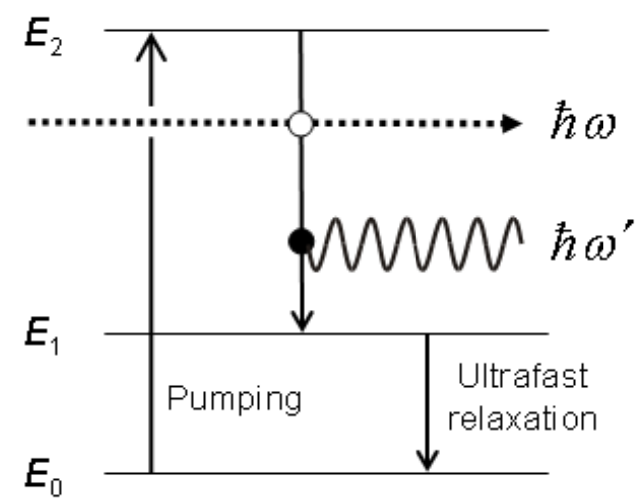

Figure 3. Energy level diagram of the three-level laser system: the wavy line represents emission $\left(\hbar \omega^{\prime}\right)$, and the off-resonant beam $(\hbar \omega)$ is the dashed arrow.

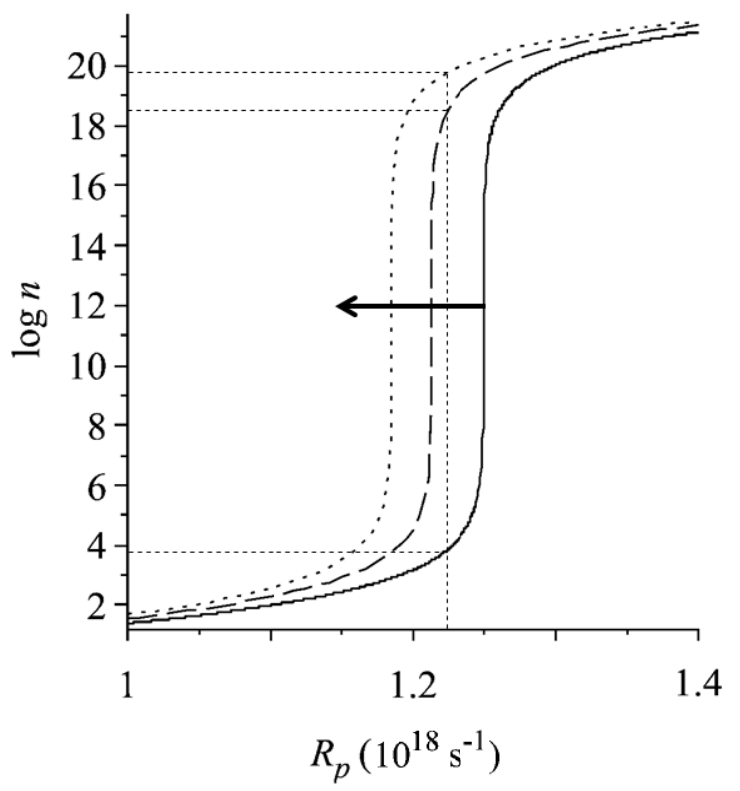

Figure 4. Plot of $\log n$, where $n$ is the number of cavity photons, against the pumping rate, $R_{p}$, for absent (solid line) and present probe beam; example irradiances of the latter are $2 \times 10^{11} \mathrm{~W} \mathrm{~cm}^{-2}$ (dashed) and $4 \times 10^{11} \mathrm{~W} \mathrm{~cm}^{-2}$ (dotted). Horizontal arrow illustrates a movement of the lasing threshold to the left for increasing laser intensities. The vertical dotted line represents a constant $R_{p}$ at 
which, on introduction of the probe beam, transistor action produces above threshold operation (denoted by the upper pair of horizontal lines).

All-optical control of such a pumped active medium is achievable by the nonlinear engagement of the laser emission with elastic forward scattering of the off-resonant probe beam (as before): the first term of equation (3) corresponds to direct emission through electronic decay between energy levels $E_{2}$ and $E_{1}$, whilst the other two terms only arise through application of the probe beam. The degree of modification of the light emission can be measured by taking the ratio of the second term - i.e. the lead correction term (as stated earlier) - against the first; the corresponding parameter $\eta$ may be approximated as:

$$
\eta=\frac{I \mu^{2}}{c \varepsilon_{0} \Delta E\left(\Delta E+\hbar \omega^{\prime}\right)} .
$$

By inspection of equation (6), it is clear that the variable $g$ will be affected on introduction of the probe beam, since the radiative and population decay rate both suffer change (but to differing degrees); the non-radiative decay rate is assumed to be constant. By simple manipulation, an expression for $g$ is given by;

$$
g(I)=1+\frac{1-Y(I)}{Y(I)+\eta Y(I)}
$$

where $Y(I)=\gamma_{\mathrm{rad}}(I) / \gamma_{2}(I)$ and $\gamma_{\mathrm{nr}} / \gamma_{2}(I)=1-Y(I)$. With the previous condition $g=5 / 4$ for $I=0$, and adopting indicative values $\mu=16 \times 10^{-30} \mathrm{C} \mathrm{m}, \Delta \mathrm{E}=10^{-20} \mathrm{~J}$ and $\hbar \omega^{\prime}=10^{-19} \mathrm{~J}$, inserting equation (8) into (6) generates the results that correspond to the two additional curves on Figure 4. For a constant pumping rate at a level indicated by the dotted vertical line, the system operates below threshold when no signal laser is present; on introduction of an off-resonant probe beam with an irradiance approaching $2 \times 10^{11} \mathrm{~W} \mathrm{~cm}^{-2}$, the output climbs by fourteen orders of magnitude, rising to sixteen orders if the signal input is doubled - typical of a laser operating at threshold. Transistor action with respect to the probe beam is clearly evident.

\section{DISCUSSION}

To achieve any all-optical device that can modify the emission properties of a suitable material using an off-resonant beam - or to modify the properties of one beam by another - is most effectively implemented by tailoring simultaneous nanoscale interactions. A photonic formulation readily identifies promising features, latent in perturbation theory, which can provide a suitable fundamental basis for such forms of action. These are terms that are normally overlooked, but which can become significant when the off-resonant beam is of sufficient intensity, or within cavity configurations that are held just below the threshold for laser action. The detailed analysis of theory thus reveals a potentially new basis for achieving all-optical transistor action, which is achievable using a relatively simple optical configuration. Compared with most other suggested schemes, this offers the advantages of ultrafast response with high repetition rate and high efficiency, with a setup that is sufficiently straightforward to invite the investigation of applications. All of the necessary theory has now been put fully in place.

\section{ACKNOWLEDGMENTS}

The authors would like to thank the Leverhulme Trust and EPSRC for funding this research. 


\section{REFERENCES}

[1] Kastrup, L., Blom, H., Eggeling, C. and Hell, S. W., "Fluorescence fluctuation spectroscopy in subdiffraction focal volumes," Phys. Rev. Lett. 94, 178104 (2005).

[2] Willig, K. I., Harke, B., Medda, R. and Hell, S. W., "STED microscopy with continuous wave beams," Nat. Methods 4, 915-918 (2007).

[3] Zhou, L. C., Liu, J. Y., Zhao, G. J., Shi, Y., Peng, X. J. and Han, K. L., "The ultrafast dynamics of near-infrared heptamethine cyanine dye in alcoholic and aprotic solvents," Chem. Phys. 333, 179-185 (2007).

[4] Armoogum, D. A., Marsh, R. J., Nicolaou, N., Mongin, O., Blanchard-Desce, M. and Bain, A. J., "Stimulated emission depletion and fluorescence correlation spectroscopy of a branched quadrupolar chromophore," Proc. SPIE 7030, 70300S (2008).

[5] Harke, B., Keller, J., Ullal, C. K., Westphal, V., Schoenle, A. and Hell, S. W., "Resolution scaling in STED microscopy," Opt. Express 16, 4154 (2008).

[6] Hell, S. W., "Microscopy and its focal switch," Nat. Methods 6, 24-32 (2009).

[7] Bradshaw, D. S. and Andrews, D. L., "Mechanism for optical enhancement and suppression of fluorescence," J. Phys. Chem. A 113, 6537-6539 (2009).

[8] Andrews, D. L. and Bradshaw, D. S., "Off-resonant activation of optical emission," Opt. Commun. 283, 43654367 (2010).

[9] Bradshaw, D. S. and Andrews, D. L., “All-optical control of molecular fluorescence," Phys. Rev. A 81, 013424 (2010).

[10] Leeder, J. M., Bradshaw, D. S. and Andrews, D. L., "Laser-controlled fluorescence in two-level systems," J. Phys. Chem. B 115, 5227-5233 (2011).

[11] Lakowicz, J. R., "Radiative decay engineering 5: metal-enhanced fluorescence and plasmon emission," Anal. Biochem. 337, 171-194 (2005).

[12] Anger, P., Bharadwaj, P. and Novotny, L., "Enhancement and quenching of single-molecule fluorescence," Phys. Rev. Lett. 96, 113002 (2006).

[13] Tam, F., Goodrich, G. P., Johnson, B. R. and Halas, N. J., "Plasmonic enhancement of molecular fluorescence," Nano Lett. 7, 496-501 (2007).

[14] Fort, E. and Grésillon, S., "Surface enhanced fluorescence,” J. Phys. D: Appl. Phys. 41, 013001 (2008).

[15] Giannini, V. and Sánchez-Gil, J. A., "Excitation and emission enhancement of single molecule fluorescence through multiple surface-plasmon resonances on metal trimer nanoantennas," Opt. Lett. 33, 899-901 (2008).

[16] Lin, C.-Y., Chiu, K.-C., Chang, C.-Y., Chang, S.-H., Guo, T.-F. and Chen, S.-J., "Surface plasmon-enhanced and quenched two-photon excited fluorescence," Opt. Express 18, 12807-12817 (2010).

[17] Lu, G., Zhang, T., Li, W., Hou, L., Liu, J. and Gong, Q., "Single-molecule spontaneous emission in the vicinity of an individual gold nanorod," J. Phys. Chem. C 115, 15822-15828 (2011).

[18] Derom, S., Berthelot, A., Pillonnet, A., Benamara, O., Jurdyc, A. M., Girard, C. and Colas des Francs, G., "Metal enhanced fluorescence in rare earth doped plasmonic core-shell nanoparticles," Nanotechnology 24, 495704 (2013).

[19] Khatua, S., Paulo, P. M. R., Yuan, H., Gupta, A., Zijlstra, P. and Orrit, M., "Resonant plasmonic enhancement of single-molecule fluorescence by individual gold nanorods," ACS Nano 8, 4440-4449 (2014).

[20] Lu, D., Kan, J. J., Fullerton, E. E. and Liu, Z., "Enhancing spontaneous emission rates of molecules using nanopatterned multilayer hyperbolic metamaterials," Nat. Nanotechnol. 9, 48-53 (2014).

[21] Andrews, D. L. and Bradshaw, D. S., "Optically tailored access to metastable electronic states," Chem. Phys. Lett. 590, 235-238 (2013).

[22] Bradshaw, D. S. and Andrews, D. L., "Laser-modified one- and two-photon absorption: Expanding the scope of optical nonlinearity,” Phys. Rev. A 88, (2013).

[23] Ford, J. S. and Andrews, D. L., "One- and two-photon absorption in solution: The effects of a passive auxiliary beam," J. Chem. Phys. 141, 034504 (2014).

[24] Bradshaw, D. S. and Andrews, D. L., "Optically controlled resonance energy transfer: Mechanism and configuration for all-optical switching," J. Chem. Phys. 128, 144506 (2008).

[25] Bradshaw, D. S. and Andrews, D. L., "All-optical switching between quantum dot nanoarrays," Superlatt. Microstruct. 47, 308-313 (2010). 
[26] Ballarini, D., De Giorgi, M., Cancellieri, E., Houdré, R., Giacobino, E., Cingolani, R., Bramati, A., Gigli, G. and Sanvitto, D., "All-optical polariton transistor," Nat. Commun. 4, 1778 (2013).

[27] Cancellieri, E., Hayat, A., Steinberg, A. M., Giacobino, E. and Bramati, A., "Ultrafast Stark-induced polaritonic switches," Phys. Rev. Lett. 112, 053601 (2014).

[28] Yanik, M. F., Fan, S., Soljačić, M. and Joannopoulos, J. D., "All-optical transistor action with bistable switching in a photonic crystal cross-waveguide geometry," Opt. Lett. 28, 2506-2508 (2003).

[29] Singh, M. R. and Lipson, R. H., "Optical switching in nonlinear photonic crystals lightly doped with nanostructures," J. Phys. B: At. Mol. Opt. Phys. 41, 015401 (2008).

[30] Chen, W., Beck, K. M., Bücker, R., Gullans, M., Lukin, M. D., Tanji-Suzuki, H. and Vuletić, V., "All-optical switch and transistor gated by one stored photon," Science 341, 768-770 (2013).

[31] Chang, D. E., Vuletic, V. and Lukin, M. D., "Quantum nonlinear optics - photon by photon," Nat. Photonics 8, 685-694 (2014).

[32] Gorniaczyk, H., Tresp, C., Schmidt, J., Fedder, H. and Hofferberth, S., "Single-photon transistor mediated by interstate Rydberg interactions," Phys. Rev. Lett. 113, 053601 (2014).

[33] Hwang, J., Pototschnig, M., Lettow, R., Zumofen, G., Renn, A., Gotzinger, S. and Sandoghdar, V., "A singlemolecule optical transistor," Nature 460, 76-80 (2009).

[34] Dawes, A. M. C., Illing, L., Clark, S. M. and Gauthier, D. J., "All-optical switching in rubidium vapor," Science 308, 672-674 (2005).

[35] Vogt, T., Viteau, M., Zhao, J., Chotia, A., Comparat, D. and Pillet, P., "Dipole blockade at Förster resonances in high resolution laser excitation of Rydberg states of cesium atoms," Phys. Rev. Lett. 97, 083003 (2006).

[36] Tiarks, D., Baur, S., Schneider, K., Dürr, S. and Rempe, G., "Single-photon transistor using a Förster resonance," Phys. Rev. Lett. 113, 053602 (2014).

[37] Siegman, A. E., [Lasers], Oxford University Press, Oxford (1986).

*david.andrews@physics.org 